\title{
Comparison of Single/Compound Plasticizer to Prepare Thermoplastic Starch in Starch-Based Packaging Composites
}

\author{
Anfu GUO ${ }^{1}$, Jianfeng $\mathrm{LI}^{2 *}$, Fangyi $\mathrm{LI}^{2}$, Jie $\mathrm{XU}^{2}$ \\ ${ }^{1}$ School of Mechanical \& Automobile Engineering, Liaocheng University, Liaocheng 252059, China \\ ${ }^{2}$ School of Mechanical Engineering, Shandong University, Jinan 250061, China \\ crossref http://dx.doi.org/10.5755/j01.ms.25.2.19143
}

Received 27 September 2017; accepted 06 March 2018

\begin{abstract}
There is a recrystallization problem with natural corn starch, the major raw material of new bio-degradable starch-based composites. To improve the adhesive properties between the starch and the fibers in composites, this paper took formamide, urea, glycerol, and glycol as plasticizers to plasticize corn starch each with the proportions (starch: plasticizer) of 10:2, 10:2.5, 10:3, 10:3.5, and 10:4. Analysis of the FTIR spectra showed that the plasticizers plasticized corn starch through the intermolecular hydrogen bonding. The results from this study showed that the hydrogen bonding between the plasticizer and the starch had the strongest bond and the plasticizing performance was the best when the proportion of starch to plasticizer was 10:3. Study of X-ray diffraction curves showed that the crystallinity of formamide-urea plasticized thermoplastic starch was $1.5 \%$, lower than that of other plasticizers. The SEM investigation showed that starch plasticization promoted a good 3-D network structure.

Keywords: thermoplastic starch, starch-based composites, plasticizer plasticizing, biomaterials.
\end{abstract}

\section{INTRODUCTION}

With increasing environmental pollution and the growing shortage of non-renewable resources, biodegradable composites [1-3] are becoming a hot research focus. Biodegradable starch-based packaging composites [4], reinforced with straw fiber, are mainly used in internal packaging, such as for dishware [5], mobile phone inner packaging, and laptop inner packaging. The major raw material of starch-based composites, natural starch by itself cannot be directly processed with thermoplastic techniques. The crystallinity of natural starch is usually $15 \%-45 \%$ [6] and when heated directly, a melting process occurs. To become more thermoplastic, its molecular structure should be disordered and the starch granules miniaturized [7,8]. With the help of a plasticizer, the glass transition temperature is lowered. Thus, the plasticity of the starch is improved. Choosing the plasticizer properly results in better plasticization performance and can effectively restrain starch retrogradation [9].

Ibrahim [1] investigated starch-based biodegradable materials made from date palm and flax fibers by plasticizing corn starch using glycerol as the plasticizer. It was found that there is a good adhesion between fibers and Glycerol Plasticized Thermoplastic Starch (GPTPS). The tensile strength can be as high as $43 \mathrm{MPa}$ when the material contains $25 \%$ each of date palm fiber and flax fiber. Guimaraes [10] studied starch-based composites, compared the cohesiveness between GPTPS and banana fibers and between GPTPS and sugarcane fibers. Plasticized starch significantly improves the mechanical properties of the composites through enforcing the adhesion between the plant fibers and starch matrix. The

*Corresponding author. Tel.: +86-531-88394593; fax: +86-53188394593. E-mail address: ljf@sdu.edu.cn (J. Li) formamide, urea, glycerol, and glycol are commonly used as starch plasticizers today $[11,12]$. There have been a number of studies on compound plasticizers. They include studies of the formamide-urea compound plasticizer [13], formamide-urea-glycerol compound plasticizer [14] and glycerol-glycol compound plasticizer $[15,16]$. It has been reported that the compound plasticizers result in better performance in plasticizing starch than the single plasticizer [17-19]. Nevertheless, to the best of the authors' knowledge, there has been no comprehensive study focusing on the reaction mechanism between the starch as the matrix and the pretreated plant fibers.

In this paper, infrared spectroscopy experiments were carried out to study the mechanism of hydrogen bonding in plasticizing thermoplastic starch (TPS) with formamide, urea, glycerol, and glycol. The plasticizing performance of compound plasticizers from the perspective of TPS crystallinity were compared by analyzing the X-ray diffraction curves. Moreover, the adhesion between the straw fibers and the TPS matrix was analyzed using the fiber-starch schematic of the water's bridge connection and SEM graphs of the composites. Finally, a homogeneous three-dimensional network structure was formed.

\section{MATERIALS AND METHODS}

\subsection{Materials and equipment}

The straw fiber was donated by Yantai Jiulong Co., Ltd. Corn starch with an average particle diameter of $70 \mathrm{~nm}$ was purchased from Hebei Huachen Starch Sugar Company Limited. The glycerol and glycol plasticizers (with a purity of $\geq 99.0 \%$ ) were purchased from Tianjin Fuyu Fine Chemical Company Limited. The urea plasticizer, with a purity of $>99.0 \%$, was also purchased from Sinopharm Chemical Reagent Company Limited. 
The formamide plasticizer, also with a purity of $>99.0 \%$, was bought from Sinopharm Chemical Reagent Company Limited. In addition, steric acid, with an assay of $98 \%$, was employed as mold-releasing agent. Talc powder, 400 mesh, was purchased from Quanzhou Xufeng powder material Co., Ltd. Sodium hydroxide $(\mathrm{NaOH})$, with a MW of 40.0, was used to treat alkalinity of the straw fibers [1].

Equipment used included the HHS-2 electronic constant temperature water bath (Shanghai Kang Road Equipment Company Limited); the JJ-1 Precision Force Electric Mixer (Changzhou Boyuan Instrument Plant); the Electronic Balance $(2000 \mathrm{~g} / 0.01 \mathrm{~g}$, Shanghai Huachao Electronic Company Limited); the thermostatic electric blast dryer oven from the Shanghai Jinghong Laboratory Instrument Company Limited; the X-ray powder diffractometer (D8 Advance, Bruker Corporation); the Fourier transform infrared spectrometer, (BRUKER VERTEX-70, Bruker Corporation); and a custom-made thermoforming mold with double columns, which functions as a hot-embossing machine.

\subsection{Fiber preparation}

The straw fibers were retted by soaking in water at $32{ }^{\circ} \mathrm{C}$ temperature for 48 hours then cut transversely to lengths of $3-5 \mathrm{~mm}$. The ratio of length to diameter of the straw fibers is about 100. Subsequently, the straw fibers were mechanically treated in a household electric blender. The fibers were then soaked in a solution $5 \% \mathrm{NaOH}$ at temperature $90{ }^{\circ} \mathrm{C}$ for $3 \mathrm{~h}$ and stirred in a household mixer for $30 \mathrm{~min}$ at a temperature ranging from $80^{\circ} \mathrm{C}$ to $90{ }^{\circ} \mathrm{C}$. To remove the separated lignin, the treated fibers were washed several times in $20^{\circ} \mathrm{C}$ water before being dipped in a solution of acetic acid ( $5 \%$ by volume) to remove from the fiber surfaces any excess $\mathrm{NaOH}$. Finally, the straw fibers were washed again in room temperature water before being dried at $100{ }^{\circ} \mathrm{C}$ for $3 \mathrm{~h}$ [1].

\subsection{Thermoplastic starch preparation}

A specific amount of corn starch (100 g) and distilled water $(300 \mathrm{~g})$ were mixed together in a beaker using a water bath with a temperature set at $75-85^{\circ} \mathrm{C}$. The proportion of plasticizer (glycerol, glycol, urea, or formamide) to starch that were each mixed together was set at 10:2, 10:2.5, 10:3, 10:3.5, and 10:4, while the proportion of the compound plasticizers (glycerol-glycol and urea-formamide) and starch were set at 1:1, 1:2, and $2: 1$. The churning time of the starch plasticization process was set to 30 minutes, since water, in particular distilled water, plays a vital role in the starch gelatinization process as the microstructure of the starch granules is fragmented when heated in water [20,21].

\subsection{Composites preparation}

The treated fibers and the thermoplastic starch matrix were put into a home-use mixer, where the polyvinyl powder (PVA, $5 \mathrm{~g})$, the stearic acid $(0.75 \mathrm{~g})$ and the talc $(15 \mathrm{~g})$ act as the binder, the release agent and the filler, respectively. Stirred uniformly at room temperature for $20-30 \mathrm{~min}$, a slurry is finally obtained. Next, we set the upper molding machine die at $185^{\circ} \mathrm{C}$ and lower die at $180{ }^{\circ} \mathrm{C}$. Next, we put $45 \mathrm{~g}$ slurry into the die and shut the die while the pressure was set to $3 \mathrm{MPa}$. Keep the pressure at $3 \mathrm{MPa}$ for $30-40 \mathrm{~s}$ and then dry for $40-45 \mathrm{~s}$ without the pressure. Finally, the composites preparation was done.

\subsection{Fourier transform infrared spectroscopy (FTIR)}

FTIR measurements of all specimens were evaluated using a BRUKER VERTEX-70 at $25^{\circ} \mathrm{C}$ wherein a pressure of $13-14 \mathrm{MPa}$ has been achieved by setting the wavelength at $400-4000 \mathrm{~cm}^{-1}$. Infrared spectra for all specimens were determined by using conventional methods. All the specimens need to be dried at a temperature of $100{ }^{\circ} \mathrm{C}$ for $12 \mathrm{~h}$ before one can proceed with grinding and mixing with $150 \mathrm{mg} \mathrm{KBr}$.

\subsection{Wide angle $X$-ray diffraction analyses}

The thermoplastic starch was dried at $100{ }^{\circ} \mathrm{C}$ for $12 \mathrm{~h}$ to get anhydrous thermoplastic starch. To grind the anhydrous thermoplastic starch thoroughly, a sieve with a 200 mesh and an agate mortar were used to prepare the sample. To make the sample flat and compacted, a sample weight of $1 \mathrm{~g}$ was added into the tank. The crystal structure of the samples was evaluated using wide angle X-Ray diffraction (conducted with the Bruker D8 Advance). The parameter settings for the machine were: $\mathrm{Cu}-\mathrm{K} \alpha$-ray; Ni filter; pipe pressure of $35 \mathrm{kV}$; pipe flow of $25 \mathrm{~mA}$; scan speed of $5 \% \mathrm{~min}$; a sampling step of $0.01^{\circ}$; and continuous scanning, repeat once [21].

\section{RESULTS AND DISCUSSION}

\subsection{Infrared spectrum analysis}

In the FTIR spectra of TPS, sharp absorption peaks associated with stretching vibrations exist around $3645-3300 \mathrm{~cm}^{-1}$. Due to the stronger hydrogen bonding between the plasticizer molecules and those of the starch, their hydrogen bonds (H-O...H) are easily formed. Hydrogen bonding effects on the infrared spectrum change the vibration frequencies of the $\mathrm{O}-\mathrm{H}$ group. The stronger hydrogen bond is, the wider the vibration spectrum of $\mathrm{O}-\mathrm{H}$ is, and the farther the spectrum moves toward lower frequencies.

From the peak values shown in Fig. 1, the O-H absorption peaks and the peak value change curves are obtained, as shown in Fig. 2.

As is shown in Fig. 2, when the ratios between the starch and the plasticizer were increased from 10:2 to $10: 3$, the absorption peaks of the O-H group moved towards lower frequencies in all four kinds of TPSs. Plasticizer molecules destroyed the original hydrogen bonds within the starch molecular structure, forming a new and stronger hydrogen bond with them. The peaks of Formamide Plasticized Thermoplastic Starch (FPTPS) changed the most, with the $\mathrm{O}-\mathrm{H}$ absorption peak moving from $56 \mathrm{~cm}^{-1}$ tow a lower frequency. This observation indicates that the intermolecular hydrogen bonding between formamide and starch is stronger than the others. When the mass ratios between the starch and the plasticizer increase from 10:3 to 10:4, the FPTPS and the Ethylene Glycol Plasticized Thermoplastic Starch 
(EGPTPS) show the smallest movement of the O-H absorption peak toward a higher frequency, followed by the Urea Plasticized Thermoplastic Starch (UPTPS) and the GPTPS.

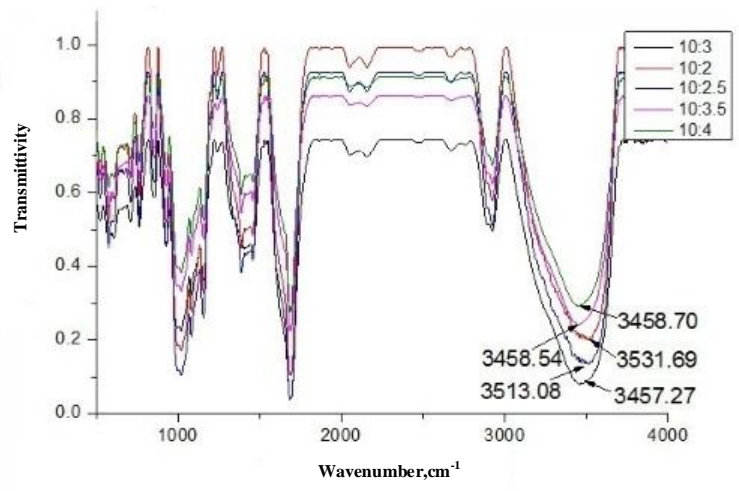

a

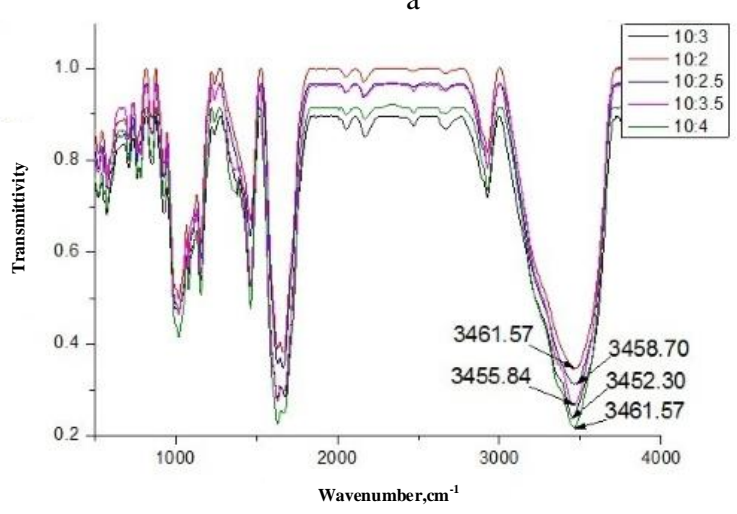

b
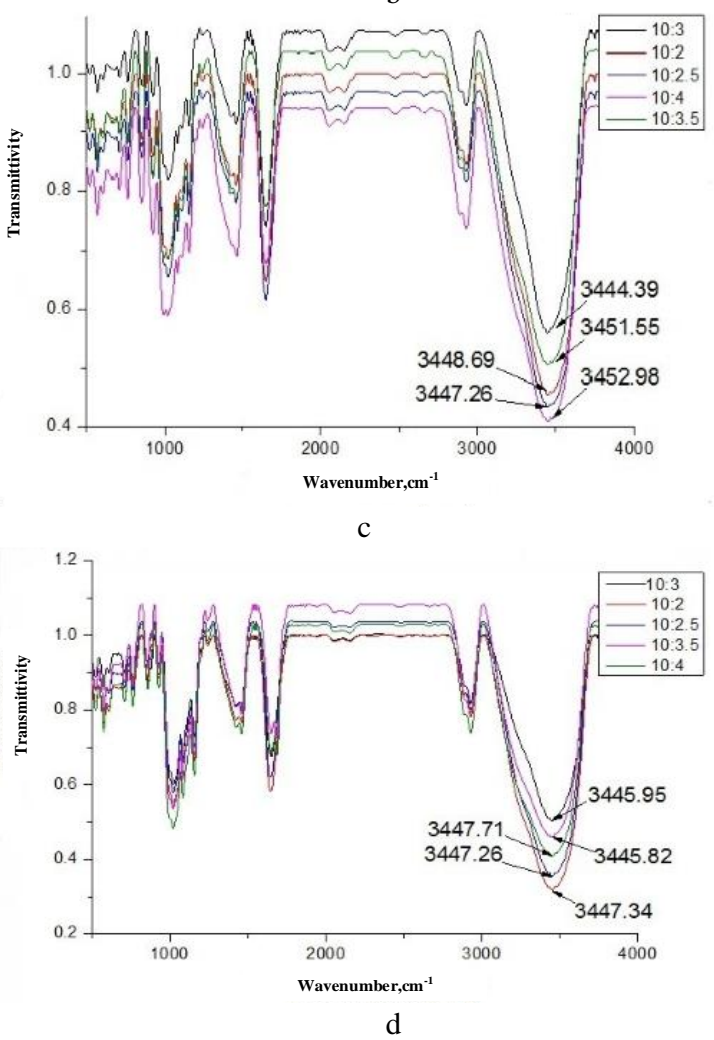

Fig. 1. FTIR spectra for four kinds of TPS (black line: mass ratio of starch to plasticizer as 10:3; red line: mass ratio as 10:2; blue line: mass ratio as 10:2.5; magenta line: mass ratio of 10:3.5; green line: mass ratio of $10: 4$ )
This result shows that when there is excess plasticizer, it will destroy the newly formed hydrogen bonds. The more damage it does to the bonds, the worse the anti-retrogradation performance the TPSs will get, which is eventually shown by a further movement of the peaks toward higher frequencies. From these results, it is concluded that FPTPS has the best anti-retrogradation performance.

In addition, there is a common pattern in the curves of all four types of TPSs. The O-H absorption peaks move towards a lower frequency first and then towards a higher frequency, which indicates that the amount of the plasticizer does make difference in the formation of hydrogen bonds with starch molecules. Only by adding a proper amount of plasticizer can good plasticizing performance be obtained. By preventing the formation of hydrogen bonds between the plasticizer and the starch, a great amount of plasticizer leads to poor plasticizing performance and forces plasticizer molecules to form hydrogen bonds within themselves. As a result, the retrogradation and the recrystallization of starch occur.

When the mass ratio of starch to plasticizer is around $10: 3$, the absorption peaks of $-\mathrm{OH}$ groups are at their minimum frequency, indicating that this is the proper ratio. At first, the peaks of the FPTPS move toward a lower frequency with the maximum displacement, then they move toward a higher frequency with the minimum displacement. The result shows that formamide forms stronger hydrogen bonds with the starch and that excessive amounts of formamide make little difference on the intermolecular hydrogen bonding, thus overcoming recrystallization of the starch by itself. This explains why the FPTPS has the best anti-retrogradation performance [9].

Additionally, the urea $(-\mathrm{NH} 2)$ in formamide and $\mathrm{NH} 2$ molecules can also form stronger hydrogen bonds along with starch molecules, which will further promote plasticizing performance. However, as determining the relevant mechanism is not straight-forward using FTIR spectra, it is beyond the scope of this paper.

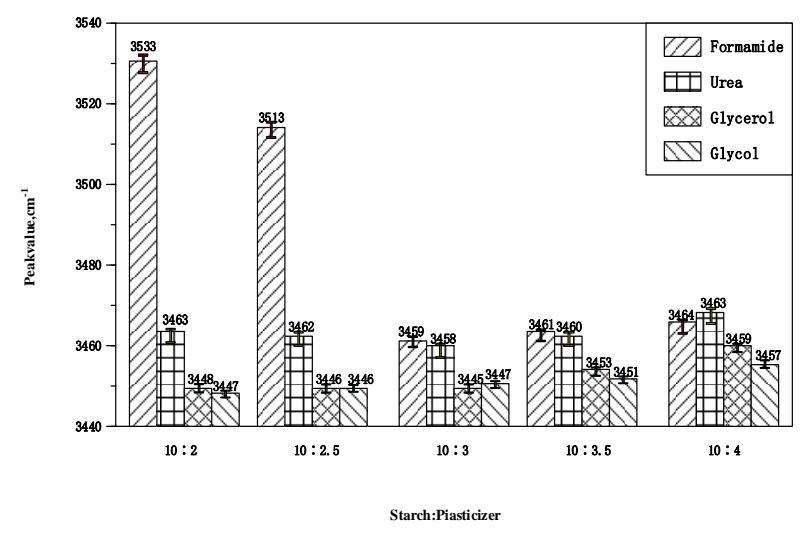

Fig. 2. O-H absorbing peaks and peak-value change

\subsection{X-ray diffraction pattern analysis}

The MDI jade5.0 software was used in this analysis. 


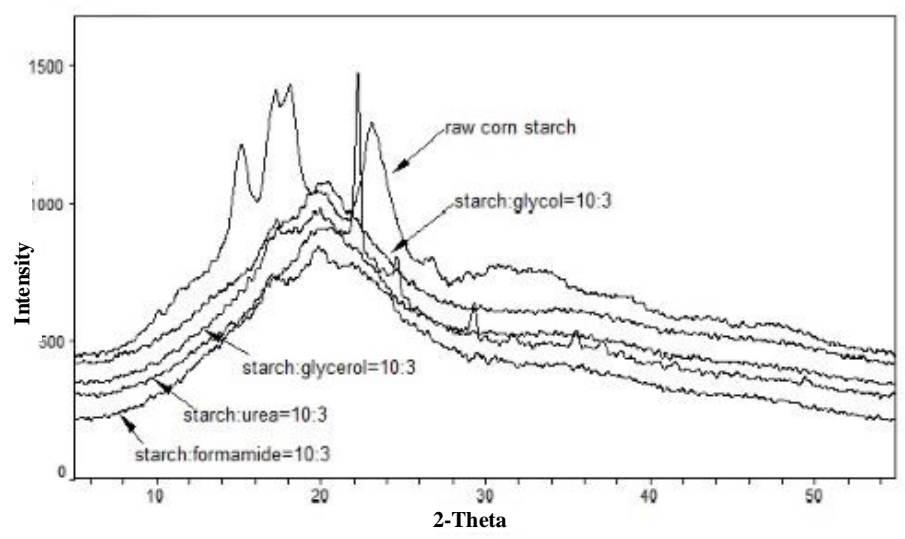

Fig. 3. XRD diffractograms when the ratio (starch to plasticizer) is $10: 3$

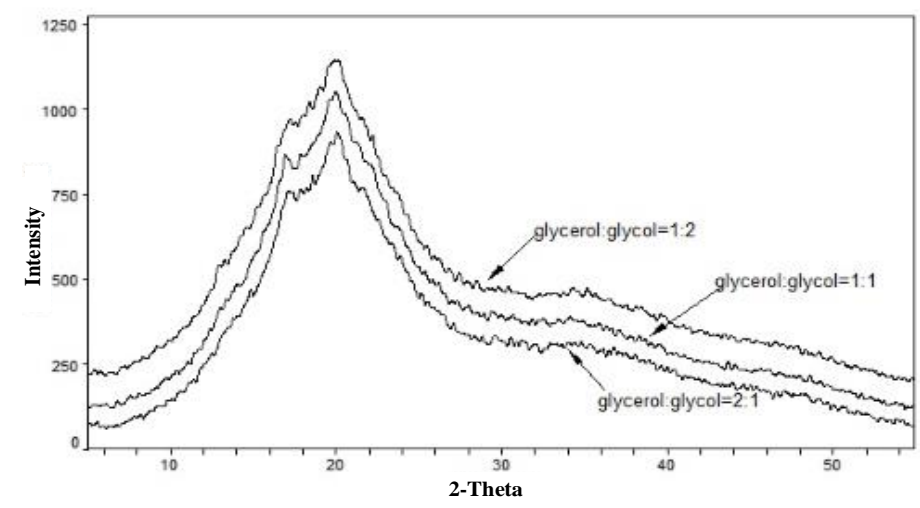

Fig. 4. XRD diffractograms of glycerol-glycol plasticized TPS

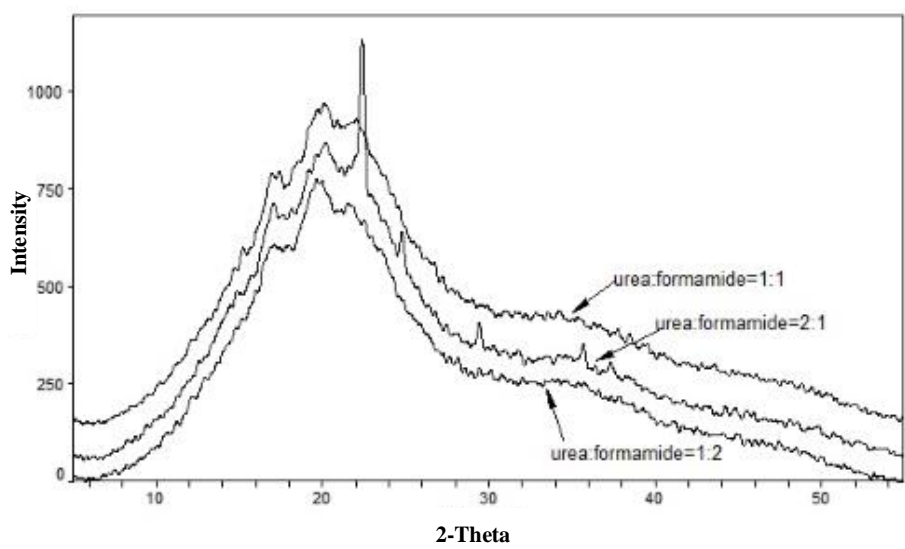

Fig. 5. XRD diffractograms of formamide-urea plasticized TPS

The XRD curves were smoothed with 11 points under extrapolated boundary conditions to eliminate the measurement errors derived from the strength of the X-ray in the raw XRD data. At a ratio of $2: 1$ of formamide to urea, the peak intensity is even lower than that of single FPTPS. The result indicates the plasticizing performance of FUPTPS is much better. It destroys the crystal structure of starch molecules to a greater degree, causing more hydrogen bonding to be formed among the starch and the plasticizers.

By fitting the peak shapes and calculating sample's crystallinity, the degree of destruction of starch molecules is quantitatively analyzed. MDI jade adopts the formula below[6]:

Crystallinity $=\frac{\text { Intensity.of } \text {.diffraction. } . \text { peak }}{\text { Total.Intensity }} \times 100 \%$.

The crystallinity of each sample is calculated and is plotted in Fig. 6.

After fitting calculation, the crystallinity of natural corn starch in the experiment is $19.93 \%$. The crystallinity of plasticized starch is dramatically decreased according to Fig. 6. According to Fig. 6 a, the formamide and the urea outperform the glycerol and the glycol in plasticizing 
starch because the electronegativity of $\mathrm{C}=\mathrm{O}$ double bonds in the formamide and the urea is higher. Thus, the electron cloud density of the oxygen atoms is higher, which makes it easier to form hydrogen bonds with hydrogen atoms in the starch. Although there are a higher number of free hydroxyl groups in the molecules' chain of glycerol and glycol, the bonding capacity of hydroxyl groups in the starch is weaker than that of formamide and urea. Thus forming stable hydrogen bonds is difficult.

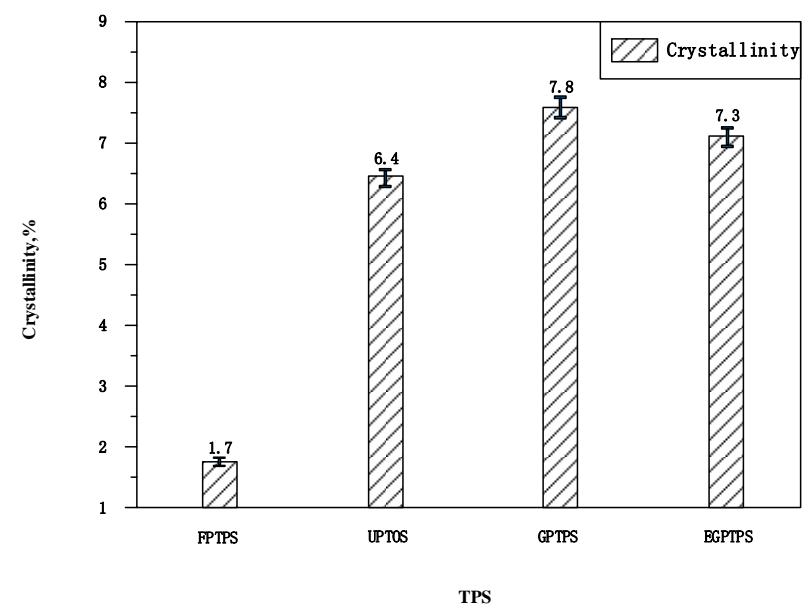

a

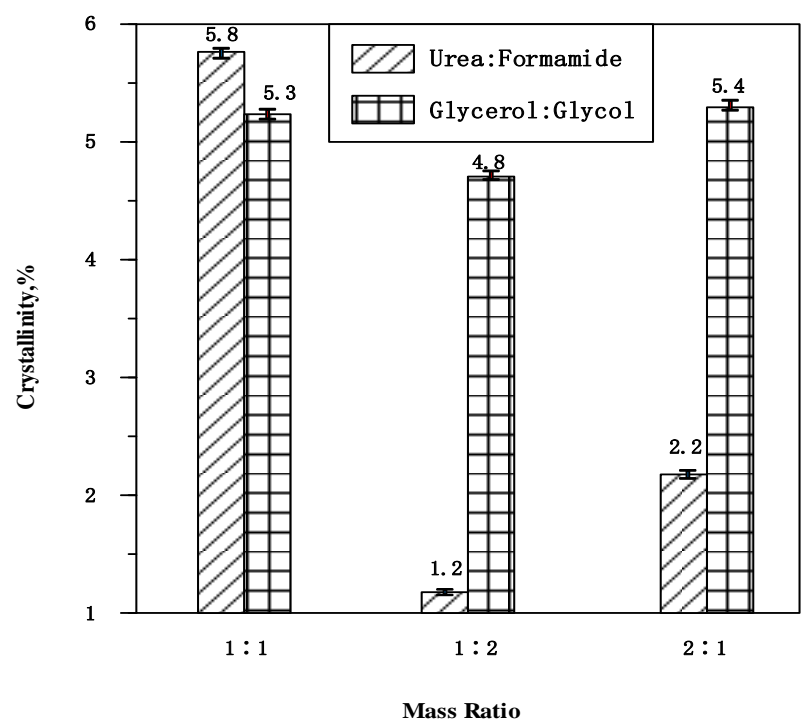

b

Fig. 6. Comparison of crystallinity: a-single plasticizer; $\mathrm{b}$-compound plasticizer (black line: urea: formamide, red line: glycerol: glycol)

According to Fig. 6 b, the compound plasticized TPS has a lower crystallinity than that of the single plasticized TPS, which also indicates a better performance of compound plasticized TPS in plasticizing and antiretrogradation. In the comparison between FUPTPS and GGPTPS, the crystallinity of FUPTPS is lower than that of GGPTPS. With a mass ratio of 1:2 between the urea and formamide, the crystallinity is the lowest at less than $1.5 \%$. This indicates that the plasticizing performance and the anti-retrogradation performance are both the best [9].

\subsection{Water's bridge-connection schematic}

A composite based solely on starch reinforced with plant fibers was prepared according to the initial process, using the introduced formamide-urea compound as the plasticizer. During the preparation of the composites, the mixture of plant fibers, the TPS, and all the additives is a solid - liquid two-phase flow in the chemical perspective and is a kind of fiber suspension. The water's bridgeconnection joining force plays a major role in the distribution of starch and fibers.

Plasticized starch aims to reduce the crystallinity of natural starch and prevents the starch retrogradation. The hydrogen bonding in the slurry is shown in Fig. 7 a. The TPS itself no longer forms hydrogen bonds, but turns to form hydrogen bonds mainly with the plasticizer. In the process of foam molding, groups exposed by the macromolecular chain of fiber are attracted to each other due to the polar traction force of water molecules. A threedimensional network structure is then formed with hydrogen bonds. Additionally, because evaporation or decomposition occurs to the plasticizer in foam molding, the exposed hydroxyl groups of TPS tend to form hydrogen bonds with the fiber molecules, as shown in Fig. 7 b. As a result, this solves the surface incompatibility problem between the fibers and the starch to some degree.

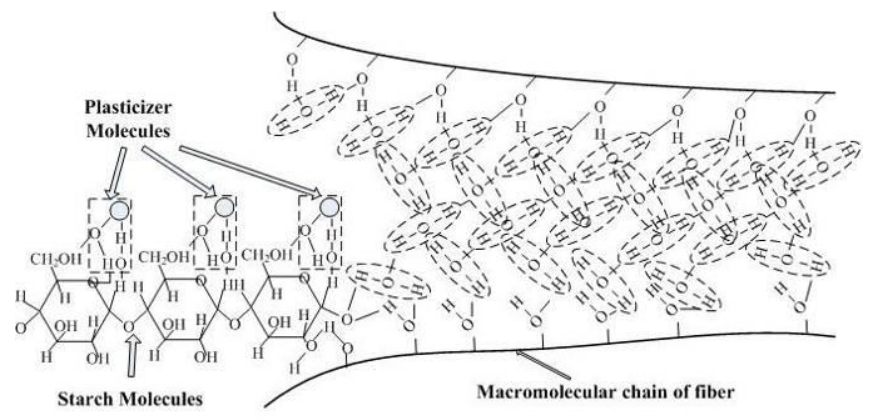

a

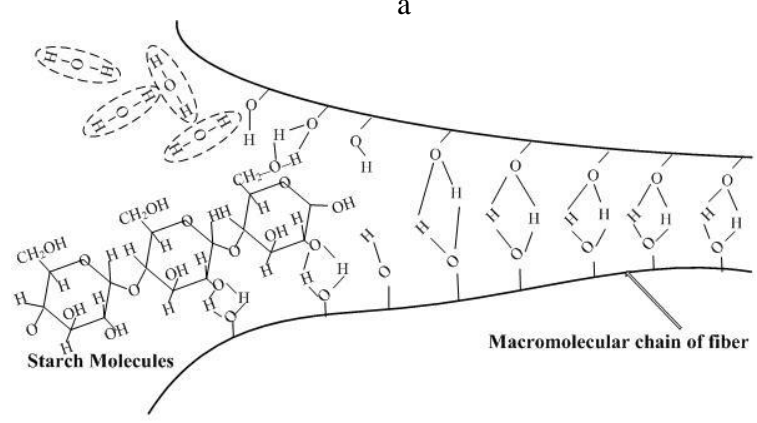

$\mathrm{b}$

Fig. 7. Schematic of water's bridge-connection of the composites: $\mathrm{a}$-hydrogen bonding in slurry; $\mathrm{b}$-hydrogen bonding in foam moulding

The fractured surfaces of the composite were evaluated with a ZEISS-SEM microscope operating at a high vacuum pressure and voltage of $20 \mathrm{kV}$. No sample preparation was done prior to imaging the surface with the SEM. The cross section SEM investigations of the composites are shown in Fig. 8. 


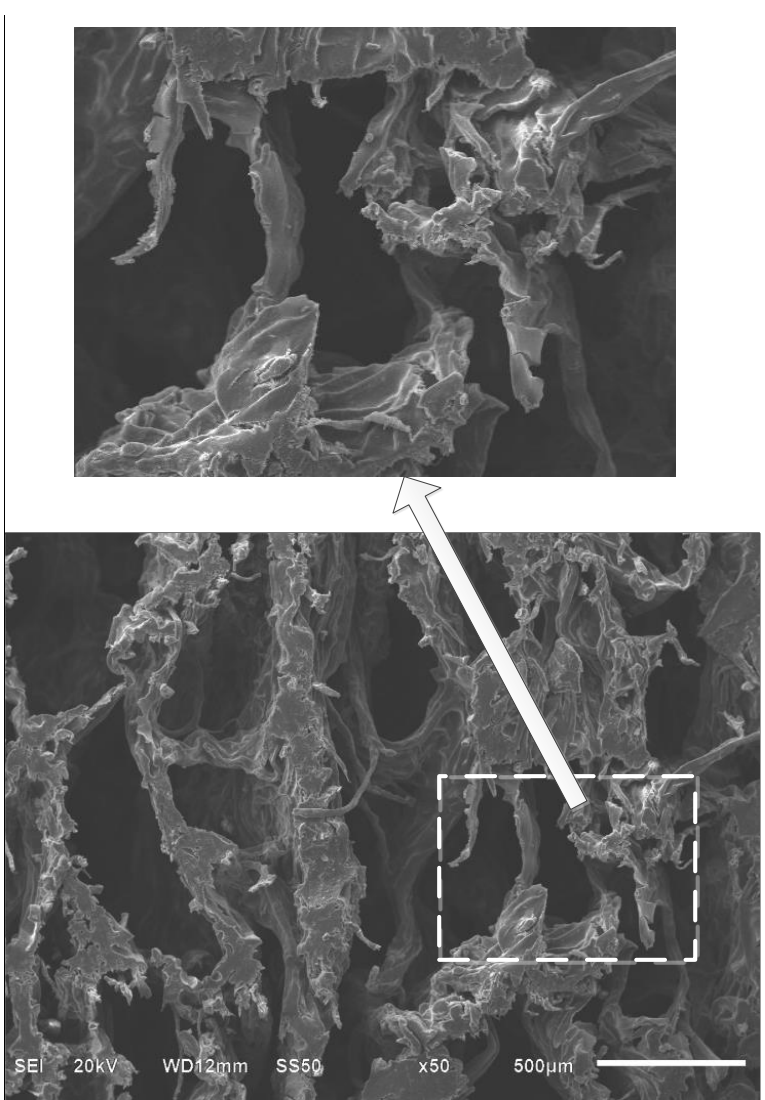

a

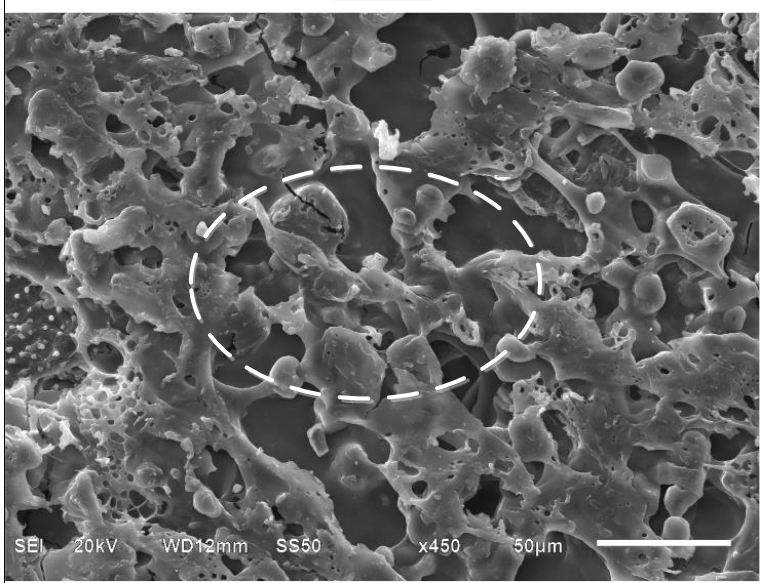

c
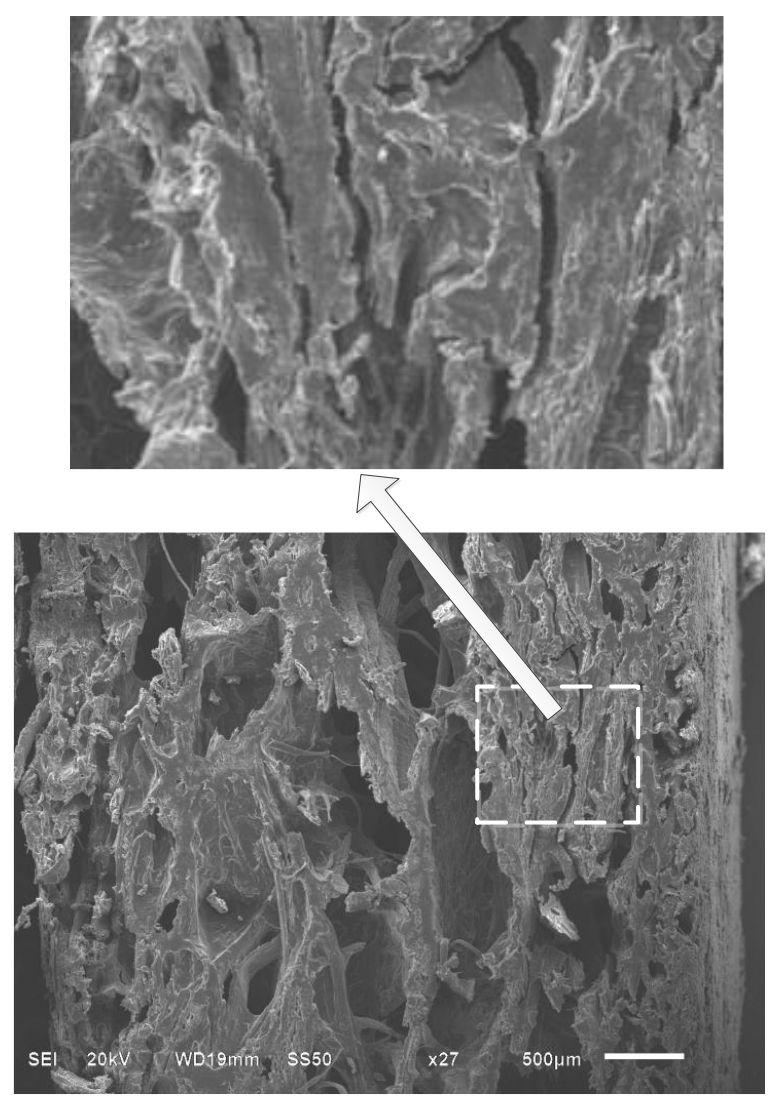

b

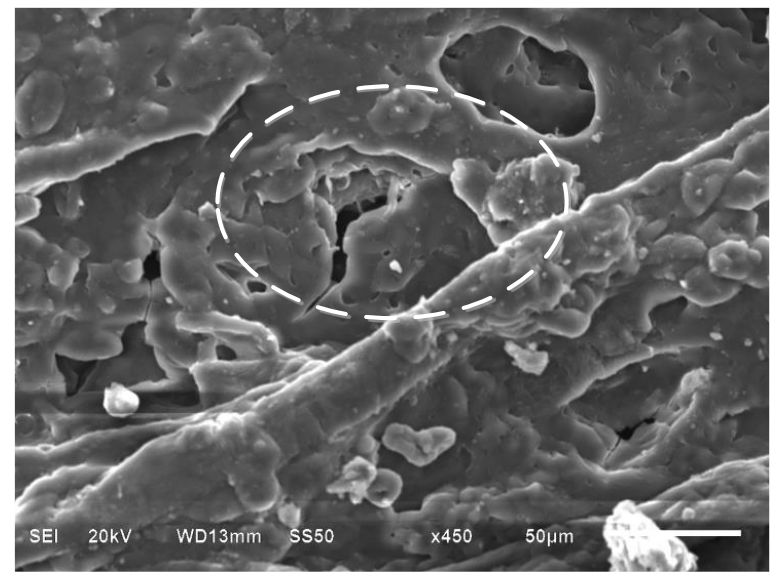

d

Fig. 8. The cross section SEM investigation of the composites: a-good fiber 3-D network structure; b-bad fiber 3-D network structure; $\mathrm{c}-$ good adhesion between fiber and starch; $\mathrm{d}$-bad adhesion between fiber and starch; a and c FUPTPS matrix composites with the ratio of formamide to urea as $2: 1 ; b$ and d GGPTPS matrix composites with the ratio of glycerol to glycol as $1: 2$

As is shown in Fig. 8 a and Fig. 8 b, the structure in Fig. 8 a is better than that in Fig. 8 b. The reason is that the surface incompatibility problem was solved and the fiber surface was covered uniformly with TPS matrix. Therefore, the fiber molecules overlapped each other and eventually formed a good fiber 3-D network structure. This ideal uniform structure provides enough room for the foaming process

\section{CONCLUSIONS}

Through infrared spectrum experiments and X-ray diffraction experiments, this paper compared the plasticizing performance of four common plasticizers (formamide, urea, glycerol, and glycol). This study showed that at a mass ratio of 10:3 (e.g., of starch to formamide), the plasticizing performance is the best for a given single plasticizer.

By analyzing the results of single plasticizers, it is concluded that the hydrogen bond (H-O...H-N) between formamide and starch is the strongest, resulting in better plasticizing performance. The minimum crystallinity of FPTPS is less than $2 \%$, which shows good antiretrogradation performance. By comparing the results of the compound plasticizers, it is shown that FUPTPS gives 
a lower crystallinity than GGPTPS. Again, with the mass ratio of $1: 2$ (e.g., urea to formamide), the crystallinity is less than $1.5 \%$, which is even lower than the $2 \%$ of single FPTPS. Hence, the FUPTPS has the best thermo plasticity and lowest crystallinity when compared to the other prepared TPSs in this paper.

The fiber-starch schematic of water's bridgeconnection and SEM investigation show that starch plasticization solves the surface incompatibility problem between plant fiber and starch, which promotes the fiber molecules to overlap each other. Eventually, the resultant structure formed a good 3-D network.

\section{ACKNOWLEDGEMENTS}

The authors gratefully appreciate the financial support from the National Natural Science Foundation of China (Nos. 51775318, 51305239 and 51275278), and Natural Science Foundation of Shandong Provincial (No. ZR2013EEQ010 and 2014ZRB019XH). We would like to thank Editage [www.editage.cn] for English language editing.

\section{REFERENCES}

1. Ibrahim, H., Farag, M., Megahed, H., Mehanny, S. Characteristics of Starch-based Biodegradable Composites Reinforced with Date Palm and Flax Fibers Carbohydrate Polymers 101 2014: pp. 11-19. https://doi.org/10.1016/j.carbpol.2013.08.051

2. Qiu, W.Y., Yu, J.W., Qiu, X.H., Deng, Z.Y., Xiao, Q. Development of Biodegradable All-starch Thermoplastics Transactions of the Chinese Society of Agricultural Engineering 24 2008: pp. 185-188. https://doi.org/10.3969/j.issn.1002-6819.2008.8.038

3. Yang, N., Sun, Z., Feng, L., Zheng, M., Chi, D., Meng, W., Hou, Z., Bai, W., Li, K. Plastic Film Mulching for Water-Efficient Agricultural Applications and Degradable Films Materials Development Research Materials and Manufacturing Processes 30 (2) 2015: pp. $143-154$.

https://doi.org/10.1080/10426914.2014.930958

4. Anfu, G., Jianfeng, L., Fangyi, L., Baokun, W. Study on the Bio-degradagility of Plant Fiber and Starch Dishware Journal of Functional Materials 40 2009: pp. 1929-1932.

5. Anfu, G., Haiyang, L., Jianfeng, L., Fangyi, L., Baokun, W., Jianyong, L., Xia, W. Mechanical Property Simulation and Verification of Plant Fiber and Starch Dishware Transactions of the Chinese Society of Agricultural Engineering 26 2010: pp. 91-95. https://doi.org/10.3969/j.issn.1002-6819.2010.07.016

6. Chen, F.Q., Zhang, B.S., Lu, H.F., Yong, Q., Zhang, X.Y. A Review of Application of X-ray Diffraction in Crystal Structure Determination of Starch Granules Food Science 31 2010: pp. 284-287.

7. López Nava, J.A., González, J.M., Chacón, X.R., Nájera Luna, J.A. Assessment of Edible Fungi and Films Bio-Based Material Simulating Expanded Polystyrene Materials and Manufacturing Processes 30 2015: pp. $1-6$. https://doi.org/10.1080/10426914.2015.1070420

8. Wu, J., Xie, B.J., Zhang, Z.H. Preparation and Characterization of Thermoplastic Micronized Starch Transactions of the Chinese Society of Agricultural Engineering 20 2004: pp. 86-88.
9. Soykeabkaew, N., Thanomsilp, C., Suwantong, O. A Review: Starch-based Composite Foams Composites Part A: Applied Science and Manufacturing 78 2015: pp. 6-263.

https://doi.org/10.1016/j.compositesa.2015.08.014

10. Guimaraes, J.L., Wypych, F., Saul, C.K. Ramos, L.P., Satyanarayana, K.G. Studies of the Processing and Characterization of Corn Starch and its Composites with Banana and Sugarcane Fibers from Brazil Carbohydrate Polymers 80 2010: pp. 130-138. https://doi.org/10.1016/j.carbpol.2009.11.002

11. Thunwall, M., Boldizar, A., Rigdahl, M. Compression and Tensile Properties of Thermoplastic Potato Starch Materials Biomacromolecules 7 2006: pp. $981-986$. https://doi.org/10.1021/bm050804c

12. Wang, J.L., Cheng, F., Zhu, P.X. Structure and Properties of Urea-plasticized Starch Films with Different Urea Contents Carbohydrate Polymers 10 2014: pp. $1109-1115$. https://doi.org/10.1016/j.carbpol.2013.10.050

13. Silva, A.G., Carter, R., Merss, F.L., Correa, D.O., Vargas, J.V., Mariano, A.B., Ordonez, J.C., Scherer, M.D. Life Cycle Assessment of Biomass Production in Microalgae Compact Photobioreactors $G C B$ Bioenergy 7 (2) 2015: pp. 184-194. https://doi.org/10.1111/gcbb.12120

14. Zuo, Y.F., Gu, J.Y., Tan, H.Y., Zhang, Y.H. Effect of Composite Plasticizer Types on Plasticization Properties of Corn Starch Journal of Functional Materials 09 2013: pp. $1365-1368$.

15. Zeng, G.S., Lin, R.Z., Zheng, L.J., Chen, L., Meng, C. Preparation and Performance Study of Waste Paper Pulp Reinforced Corn Starch-based Composites Journal of Functional Materials 16 2012: pp. 2218-2221.

16. Gang, L., Fangyi, L., Kaikai, G., Peng, L., Yu, L., Xiujie, J., Jianfeng, L. Preparation and Properties of Biomass Cushion Packaging Material Journal of Functional Materials 13 2013: pp. 1969-1973.

17. Rosa, M.F., Chiou, B., Medeiros, E.S., Wood, D.F., Williams, T.G., Mattoso, L.H., Orts, W.J., Imam, S.H. Effect of Fiber Treatments on Tensile and Thermal Properties of Starch/ethylenevinyl Alcohol Copolymers/coir Biocomposites Bioresource Technology 100 2009: pp. 5196-5202. https://doi.org/10.1016/j.biortech.2009.03.085

18. Wang, Y.H., Liu, M.R., Gao, Z.D., Li, G.Z. Influence of Alkali Solution on Mechanical Property of Plant Fiber Gypsum Composites Wall Materials Innovation \& Energy Saving in Buildings 11 2009: pp. 38-41.

19. Goriparthi, B.K., Suman, K.N.S., Rao, N.M. Effect of Fiber Surface Treatments on Mechanical and Abrasive Wear Performance of Polylactide/jute Composites Composites: Part A 3 2012: pp. 1800-1808. https://doi.org/10.1016/j.compositesa.2012.05.007

20. Fu, Z.Q., Wang, L.J., Zhou, H., Li, D., Adhikari, B. Studies on the Starch-water Interactions Between Partially Gelatinized Corn Starch and Water during Gelatinization Carbohydrate Polymers 101 2014: pp. 727-732. https://doi.org/10.1016/j.carbpol.2013.09.098

21. Chengzhao, W., Fangyi, L., Liming, W., Anfu, G., Jianfeng, L., Chuanwei, Z., Peng, L. Research on Thermoplastic Starch and Different Fiber Reinforced Biomass Composites RSC Advances 5 2015: pp. 49824-49830. https://doi.org/10.1039/c5ra08278 